\title{
HIV Counseling and Testing of Psychiatric Patients: Time to Reexamine Policy and Practices
}

\author{
David G. Ostrow, M.D., Ph.D.
}

In the current issue of General Hospital Psychiatry, Drs. Hellerstein and Prager document the lack of attention to human immunodeficiency virus (HIV) risk assessment and counseling for patients undergoing intake evaluation in a general hospital psychiatric clinic [1]. That this was the case among patients being seen at a major HIV/AIDS treating hospital in New York City only serves to underline the need to integrate HIV-related health care into mainstream medical and mental health care systems. Examining a group of acute psychiatric inpatients at another NYC hospital, Sacks et al. [2] recently documented a relatively high rate of HIV behavioral risk (19\%) that was associated with a lack of knowledge or concern about HIV infection among a majority of the at-risk patients. These and other studies, taken together, suggest that though psychiatric patients may have a high prevalence of HIV infection and risk behaviors, they are not being adequately assessed or counseled for HIVrelated behavioral, affective, or cognitive problems in the course of their psychiatric assessment. If this is so in the city with the largest number of persons with AIDS (PWAs) in the U.S., we can only imagine the situation elsewhere.

Meanwhile, a number of advances in our understanding of the epidemiology, prevention, and treatment of HIV infection makes it necessary to reexamine our present policies and practices concerning the HIV counseling and testing of psychiatric patients. These advances include the in-

Midwest AIDS Biobehavioral Research Center, University of Michigan School of Medicine, Institute for Social Research, Ann Arbor, Michigan.

Address reprint requests to: David G. Ustrow, M.D., Ph.D. Room 5030, Institute for Social Research, 426 Thompson St., Ann Arbor, MI 48106-1248. creasing impact of HIV on women, racial and ethnic minorities, and heterosexual partners of the "traditional" AIDS risk group members (i.e., bisexual men and intravenous drug users); the recognition that early treatment with anti-retrovirals and prophylactic agents for the most common opportunistic infections can reduce the likelihood of persons living with HIV (PWHs) developing AIDS; and a growing appreciation of the fact that the majority of PWHs and persons at risk for infection are unaware of that infection or risk. Thus, psychiatric facilities may be ideally suited to identifying persons at risk for HIV infection and initiating appropriate preventive measures, early intervention counseling, and treatment. On the basis of such developments, Dr. James Strain [3] recently argued that it is time to implement mandatory HIV counseling and testing for all psychiatric inpatients.

In the same issue of Psychiatric News, Dr. Marshall Forstein presented the major arguments against mandatory or even routine HIV testing of psychiatric patients. These arguments included the issue of false-positive tests and cost-benefit considerations, both of which are likely to become less important as testing and treatment advances increase the accuracy and benefits of HIV screening. Other arguments against mandatory or routine testing, however, remain compelling: limited access to early HIV interventions and the medical indigency levels of the mental health patient population means that the persons most likely identified as HIV-infected are those least likely to benefit from that knowledge and most likely to suffer from discrimination; the inadequacy of our mental health care system to provide adequate counseling and confidentiality to persons identified as HIV seropositive; and the barriers to treatment and to the fostering of the patient-therapist 
relationship that any form of mandatory testing would present.

Though these issues are likely to remain pertinent for the foreseeable future, especially in the current climate of reduced resources for mental health care, we agree with Dr. Strain when he says that "stigmatization and bias are insufficient reasons to not examine the evidence, analyze the data, and promulgate guidelines based on the best available science" [3]. Such an examination will most certainly lead to two important conclusions. First, our knowledge of the mental health consequences of HIV infection and the AIDS epidemic remains woefully inadequate, despite significant amounts of AIDS mental health research during the past 5 years. What is known about the prevalence, incidence, and treatment of mental illness in PWH $\mathrm{P}$ PWAs comes largely from studies of relatively healthy and advantaged gay and bisexual men [4] and may not be applicable to the majority of persons identified through HIV screening of psychiatric patients. And second, HIV assessment and counseling among psychiatric patients are seriously inadequate, despite the recommendations of the Centers for Disease Control (CDC) and the APA, and despite significant efforts on the part of the NIMH, APA, the American Psychological Association, and many other professional associations to educate mental health care providers. These conclusions argue strongly for increased attention to the mental health consequences of HIV infection and the efficacy of different models of counseling, testing, and mental health care delivery on those problems. But we are still left with the nagging sense that we can and should be doing more right now while we are awaiting better data, as the public health imperatives of the epidemic mandate proactive approaches that take advantage of opportunities to identify, counsel, and treat all persons with HIV infection.

As long as there are significant ethical and practical arguments against mandatory or routine HIV testing of psychiatric patients, what are the most prudent and beneficial policy and practice recommendations that can be made? Why not actually implement the current APA and CDC recommendations, recommendations not now being effectively carried out? As the studies of Hellerstein and
Prager and others make clear, these measures would include making HIV risk assessment and counseling (including an explanation of the rationale for testing) a mandatory part of all psychiatric evaluations; making AIDS/HIV education of all mental health care givers, including training in infection control procedures and risk assessment/ counseling skills, mandatory for licensing and certification; increasing confidentiality provisions while making documentation of risk assessment and counseling a required part of intake evaluations; funding by NIMH and HRSA of model programs for HIV-related mental health care delivery (ensuring that they are applicable to the realities of mental healthcare); and lobbying, through both our national professional organizations and local mechanisms, for passage of antidiscrimination legislation and complete funding of the Ryan White CARES legislation, the latter of which would provide early HIV intervention and AIDS treatment for those most likely to be identified through HIV counseling and testing of psychiatric patients.

We have a long way to go before we have $100 \%$ effective and compassionate treatments for the psychosocial, behavioral, and neuropsychiatric problems engendered by the AIDS epidemic. But, we also have a rapidly growing body of knowledge that can be effectively put to use if we set aside our fears and embrace the mental health problems of PWHs as the ideal challenge to modern biopsychosocial psychiatry.

\section{References}

1. Hellerstein DJ, Prager ME: Assessing HIV risk in the general hospital psychiatric clinic. Gen Hosp Psychiatry, $14: 3-6,1992$

2. Sacks MH, Silberstein C, Weiler P, Perry S: HIVrelated risk factors in acute psychiatric inpatients. Hosp Community Psychiatry 41:449-451, 1990

3. Strain J, Forstein $\mathrm{M}$ : 'Yes' and 'no'. ViewpointsCrossfire: Is it time to require mandatory HIV testing of all hospitalized inpatients. Psychiatric News, March 15, 1991, pp 9-30

4. Williams JBW, Rabkin JG, Remien RH, Gorman JM, Ehrhardt AA: Multidisciplinary baseline assessment of homosexual men with and without human immunodeficiency virus infection. II. Standardized clinical assessment of current and lifetime psychopathology. Arch Gen Psychiatry 48:124-130, 1991. 